\title{
Low-Complexity Digital Tone Detector for Telecommunications Switching Systems
}

\author{
VITO CAPPELLINI, MEMBER, IEEE, AND ENRICO DEL RE, MEMBER, IEEE
}

\begin{abstract}
An improved structure for an envelope detection multifrequency receiver based on digital nonrecursive quadrature filters is proposed. The receiver utilizes a property of digital nonrecursive quadrature filters to reduce the required computational complexity by a nominal factor of 2 with a negligible hardware increase. The application to the tone

Manuscript received July 26, 1982; revised March 15, 1983. This work was supported by C.N.R. (National Research Council, Italy) under Grant 82.01417.07. An earlier version of this paper was presented at GLOBECOM '82, Miami, FL, November 29-December 2, 1982.

$\mathrm{V}$. Cappellini is with the Dipartimento di Ingegneria Elettronica, Università di Firenze and I.R.O.E.-C.N.R., 50139 Florence, Italy.

E. Del $\mathrm{Re}$ is with the Dipartimento di Ingegneria Elettronica, Università di Firenze, 50139 Florence, Italy.
\end{abstract}

detector implementation for two CCITT standard signaling systems is considered, showing the low computational and structural complexity required by the proposed technique. The required multiplication rate is reduced by more than twice with respect to other conventional approaches.

\section{INTRODUCTION}

$\mathrm{T}$ ELECOMMUNICATIONS switching systems commonly use multifrequency tone transmissions for the signaling operations. Bursts of one or more frequencies are employed to encode the information to be transmitted and, generally, noncoherent detection is preferred at the receiver 
because it attains the required system performance with an acceptable implementation complexity.

Among the approaches proposed for a digital multifrequency receiver, including bandpass filtering [1], [2], use of the discrete Fourier transform [3], [4], tone parameter extraction [5], pattern recognition detection [6], and envelope detection [7]-[10], the latter solution appears one of the most promising and attractive methods from a practical implementation point of view. It can be implemented, for example, by multiplying the receiver waveform with two in-quadrature sinusoids and subsequent low-pass filtering [7] or by using two bandpass filters with a 90 degree phase shift difference (quadrature filters) [8]-[10].

Here we present an improved structure of an envelope detection multifrequency receiver based on digital quadrature filters. In Section II the basic operations of a quadrature detection receiver are briefly recalled, pointing out in particular the advantages of using finite impulse response (FIR) digital filters. In Section III the proposed receiver structure based on the properties of digital FIR quadrature filters [11] is presented. In Section IV the design of a tone detector for the standard signaling systems CCITT Nos. 5 and R1 is described, showing that the required multiplication rate is reduced by more than twice with respect to a straightforward solution to the quadrature tone detection and by more than six times with respect to a conventional bandpass approach.

\section{Quadrature Detection}

The envelope detection by quadrature filtering has the advantage of avoiding any demodulation process at the receiver, with respect to the more conventional technique of multiplying the received signal with inquadrature sinusoids and subsequent low-pass filtering [7].

The quadrature detection of one or more among $M$ possible frequencies $f_{i}, i=1, \cdots, M$, can be realized by supplying the received waveform to $M$ couples of quadrature filters $H_{i}(f)$ and $\hat{H}_{i}(f)$ able to produce $M$ Hilbert transform pairs, $x_{i}(t)$ and $\hat{x}_{i}(t)$, respectively, at their outputs. The filters $H_{i}(f)$ are nonoverlapping bandpass filters of sufficiently narrow bandwidth $W$, whose respective center frequency coincides with the nominal frequency $f_{i}$ of a different transmission tone and the filters $\hat{H}_{i}(f)$ are defined as

$$
\hat{H}_{i}(f)=-j \operatorname{sgn} f H_{i}(f)
$$

being $j=\sqrt{-1}$. The envelope of the $i$ th transmission frequency can be estimated as

$$
a_{i}(t)=\left[x_{i}^{2}(t)+\hat{x}_{i}^{2}(t)\right]^{1 / 2}
$$

which in ideal conditions (i.e., absence of interferences and noise and ideal filter frequency masks) should be a constant value. An equivalent detection strategy is the evaluation of

$$
r_{i}(t)=a_{i}^{2}(t)
$$

that avoids any square root operation in the receiver implementation. The statistical performance of a receiver of this type has been analyzed in [12] and a preliminary receiver prototype using microprocessor-controlled CCD-based FIR quadrature filters was described in [9].

As the envelope is a low-pass signal bandlimited to $W / 2$, the filtering operations involved in the envelope or envelope square evaluation may be performed only once every $1 / W$ seconds since it suffices to compute the quantities

$$
r_{i}(n / W)=x_{i}^{2}(n / W)+\hat{x}_{i}^{2}(n / W)
$$

for $n$ integer and $i=1, \cdots, M$.

This is particularly advantageous for an FIR digital realization of the filters $H_{i}(f)$ and $\hat{H}_{i}(f)$ that may supply the samples of their outputs directly at the reduced rate $W$. This operation permits the time multiplexing of a single hardware filtering unit among all the filters to be implemented [9].

Furthermore, if the transmission frequencies satisfy certain conditions, the structure and the computational complexity of a digital quadrature detection multifrequency receiver can benefit from a property of digital quadrature filters, as illustrated in the following section.

\section{Receiver Structure}

FIR linear-phase digital quadrature filters of even length $N$ have the property [11] that the impulse responses $f(n)$ and $g(n)$ of the two filters

$$
\begin{aligned}
& F(f)=A(f) e^{-j \pi f(N-1) T}, \quad|f| \leqslant \frac{1}{T} \\
& G(f)=-j \operatorname{sgn} f A\left(f-\frac{1}{2 T}\right) e^{-j \pi f(N-1) T}, \quad|f| \leqslant \frac{1}{T}
\end{aligned}
$$

$1 / T$ being their associated sampling rate, are simply related by

$$
g(n)= \pm(-1)^{n} f(n), \quad 0 \leqslant n \leqslant N-1
$$

where + is for $N=2(2 k)$ and - is for $N=2(2 k+1)$, with $k$ any integer. The filter $G(f)$ is the quadrature filter associated to the linear-phase filter whose magnitude response is the symmetric one of that of $F(f)$ with respect to $1 / 4 T$. The two filters $F(f)$ and $G(f)$ have their impulse responses simply related by an alternate sign inversion operation.

If the $M$ possible transmission frequencies $f_{i}, i=$ $1, \cdots, M$ ( $M$ even) satisfy the relation

$$
f_{M-i+1}=1 / 2 T-f_{i}, \quad i=1, \cdots, M
$$

$1 / T$ being the sampling rate of the signal digital filtering inside the multifrequency receiver, the couples of quadrature filters $H_{i}(f), \hat{H}_{i}(f)$, and $H_{M-i+1}(f), \hat{H}_{M-i+1}(f)$ are symmetric with respect to $1 / 4 T$ and, if they are realized by FIR linear-phase digital filters of even length $N$, according 


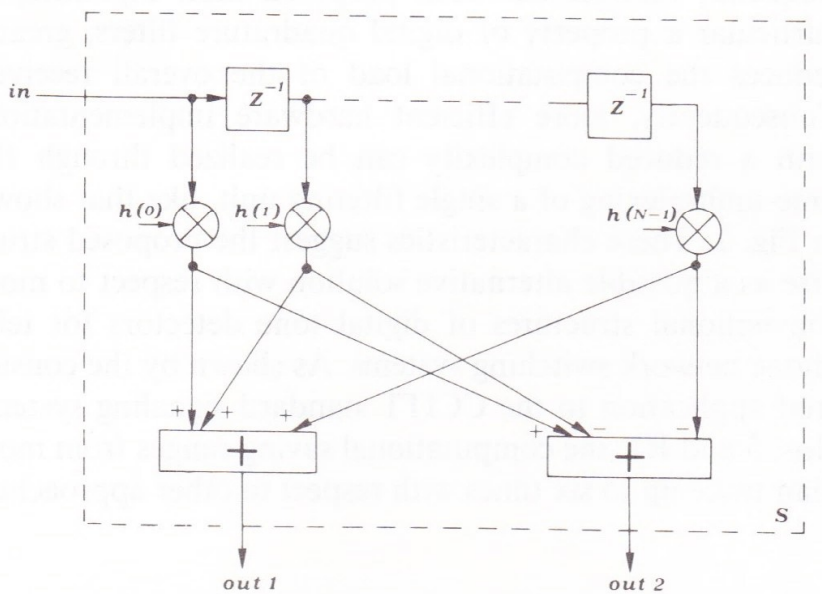

Fig. 1. Filtering structure exploiting the property of digital quadrature FIR filters.

to (4), (5), and (6), their impulse responses are in couples related by

$$
\begin{aligned}
& \hat{h}_{M-i+1}(n)= \pm(-1)^{n} h_{i}(n) \\
& h_{M-i+1}(n)= \pm(-1)^{n} \hat{h}_{i}(n), \quad i=1, \cdots, M .
\end{aligned}
$$

According to the properties (8), the filtering structure of Fig. 1 supplies two different signals (out 1 and out 2) to the quadrature envelope detector essentially through the computational complexity of only one filtering operation. It only needs an additional adder to the classical FIR filter structure realized in the direct form. When in Fig. 1

$$
h(n)=h_{i}(n), \quad i=1, \cdots, M
$$

out 1 supplies $x_{i}(k T)$ and out 2 supplies $\pm \hat{x}_{M-i+1}(k T)$, $k$ integer, while when

$$
h(n)=\hat{h}_{i}(n), \quad i=1, \cdots, M
$$

out 1 supplies $\hat{x}_{i}(k T)$ and out 2 supplies $\pm x_{M-i+1}(k T)$, the sign depending on the filter length. The sign uncertainty can, of course, be removed, but is inessential in the present application due to the square operations in (2). In the time interval $T$ between two consecutive signal samples, the switching of the two sets of filter coefficients $h_{i}(n)$ and $\hat{h}_{i}(n), 0 \leqslant n \leqslant N-1$, for a specific $i$, is sufficient to supply the four quantities $x_{i}(k T), \hat{x}_{i}(k T), x_{M-i+1}(k T)$, and $\hat{x}_{M-i+1}(k T)$ necessary for the envelope evaluations at the frequencies $f_{i}$ and $f_{M-i+1}$ at the time instant $t=k T$ according to (2) or (3).

According to (3a), the single hardware filtering unit of Fig. 1 can be time-multiplexed among more than two couples of quadrature filters if the switching and filtering operations are sufficiently fast. As a limiting situation, all the $2 M$ filterings can be performed through the switching of the corresponding $M$ sets of filter coefficients. Intermediate hardware implementations are possible by using few hardware filtering units to preform all the $2 M$ required filterings.

The hardware structure of Fig. 1 performs the $2 M$ quadrature detection filterings essentially through the com-

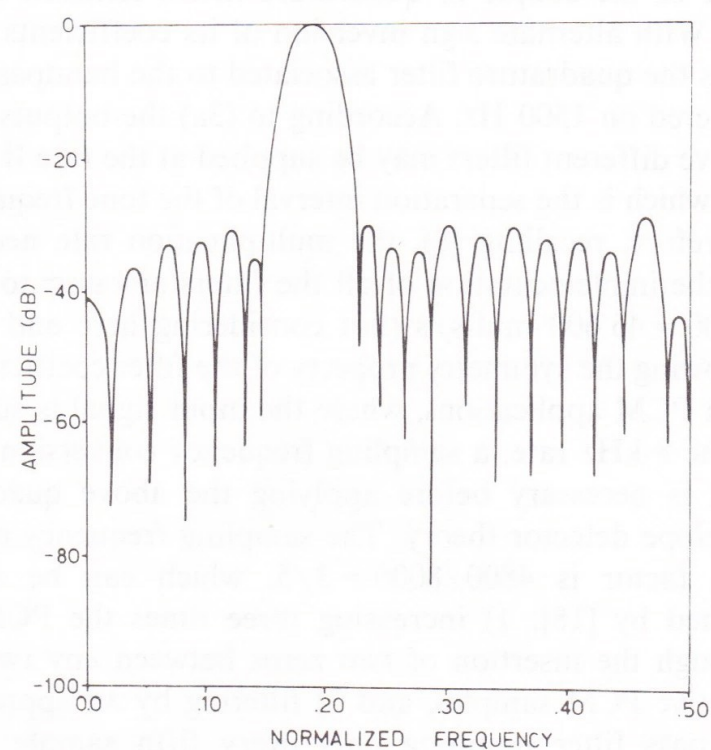

Fig. 2. Frequency response of the FIR bandpass filter centered on 900 $\mathrm{Hz}$ with 38 coefficients rounded to 8 bits. By alternate sign inversion of its coefficients it also gives the quadrature filter associated to the bandpass filter centered on $1500 \mathrm{~Hz}$

putational load of $M$ filterings (thus increasing the receiver speed by a nominal factor of 2 ).

Classical design techniques [17] lead to real FIR digital quadrature filters that do not have their passband ripples aligned. It is a straightforward matter to show that this misalignment produces a time-varying envelope square estimate in the range $1 \pm 2 \delta$ times the true value, being $\delta \ll 1$ the maximum passband ripple. When this effect could not be tolerated, the design of complex FIR filters can be carried out [13]-[14]. The real and imaginary parts of their impulse response synthesize quadrature filters with perfectly aligned masks.

\section{A Case Study: Application to CCitT Nos. 5 And R1 Signaling Systems}

As a particular application example the design of a digital tone detector for the CCITT signaling systems Nos. 5 and R1 [15], [16] is considered here. For register signaling they employ a two-out-of-six frequency code. The prescribed nominal frequencies are 700, 900, 1100, 1300, 1500, and $1700 \mathrm{~Hz}$, with a duration ranging from several tens of ms up to $100 \mathrm{~ms}$.

To apply the above theory, the sampling frequency of the digital quadrature filters must be equal to $4800 \mathrm{~Hz}$. Six couples of quadrature filters are required for the envelope detection of each tone. Of course, according to relations (8), with $M=6$, only six different real filters or three different complex filters need to be actually designed. Real filters of the equiripple type were chosen and designed by the computer program based on the Remez exchange algorithm [17]. The frequency specifications imposed by the CCITT requirements are met by FIR filters of length $N=38$, with coefficients rounded to 8 bits. Fig. 2 shows, as an example, the frequency response of designed bandpass 
filter of the couple of quadrature filters centered on 900 $\mathrm{Hz}$. With alternate sign inversion of its coefficients it also gives the quadrature filter associated to the bandpass filter centered on $1500 \mathrm{~Hz}$. According to (3a) the outputs of the twelve different filters may be supplied at the rate $W=200$ $\mathrm{Hz}$, which is the separation interval of the tone frequencies. Therefore, recalling (8), the multiplication rate necessary for the implementation of all the filters amounts to $6 \times 38$ $\times 200=45600$ mults $/ \mathrm{s}$ (not considering here and in the following the symmetry property of the filter coefficients).

In PCM applications, where the input signal is supplied at the $8 \mathrm{kHz}$ rate, a sampling frequency conversion to 4.8 $\mathrm{kHz}$ is necessary before applying the above quadrature envelope detector theory. The sampling frequency conversion factor is $4800 / 8000=3 / 5$, which can be accomplished by [18]: 1) increasing three times the PCM rate through the insertion of two zeros between any two consecutive PCM samples, and 2) filtering by an appropriate low-pass filter retaining only every fifth sample at the output. The focal point of the sampling rate conversion is the design of the low-pass filter operating at $24 \mathrm{kHz}$ that has to attenuate to a sufficient degree the baseband signal replicas in order to prevent aliasing in the decimation process. An FIR design is a convenient choice for such a low-pass filter. Considering the frequency range occupied by the possible transmission tones and the CCITT operating specifications, an equiripple FIR filter of length 9 is sufficient with coefficients rounded to 8 bits. To produce the desired output, it needs $9 \times 4800 / 3$ mults $/ \mathrm{s}$. Hence, the overall tone detector computational complexity amounts to 60000 mults/s.

The absolute delay introduced by the sampling rate conversion filter is $\frac{1}{2}(9-1) / 24 \mathrm{~ms}=0.1667 \mathrm{~ms}$, and the transient response of the quadrature FIR filters is $\frac{1}{2}(38-$ 1) $/ 4.8 \mathrm{~ms}=3.854 \mathrm{~ms}$. Their sum $(\sim 4 \mathrm{~ms})$ is small compared to the tone duration. Therefore, the time response of the filters assures a correct receiver operation for most of the time.

As a comparison, the multiplication rate of a quadrature envelope detector directly operating on the $8 \mathrm{kHz} \mathrm{PCM}$ signal would be $12 \times 60 \times 200=144000$ mults $/$ s, requiring at least FIR filters of length 60 to achieve comparable tone detector performance: indeed, in [10] FIR filters of length 64 are proposed. The solution based on demodulation of the incoming signal followed by digital IIR low-pass filtering, presented in [7], needs 240000 mults/s. Other approaches based on bandpass filtering of the received tones have even greater multiplication rates because they must supply the output signals at the $8 \mathrm{kHz}$ frequency of the input signal, even though six bandpass filterings are now sufficient: for example, the system proposed in [2], employing six infinite impulse response fourth-order bandpass filters operating at $8 \mathrm{kHz}$, requires 384000 mults/s. frequency receiver has been proposed that, exploiting in particular a property of digital quadrature filters, greatly reduces the computational load of the overall receiver. Consequently, more efficient hardware implementations with a reduced complexity can be realized through the time-multiplexing of a single filtering unit, like that shown in Fig. 1. These characteristics suggest the proposed structure as a possible alternative solution with respect to more conventional structures of digital tone detectors for telephone network switching systems. As shown by the considered application to the CCITT standard signaling systems Nos. 5 and R1, the computational saving ranges from more than twice up to six times with respect to other approaches.

\section{REFERENCES}

[1] B. Gopinath and R. P. Kurshan, "A touch-tone receiver-generator with digital channel filters," Bell Syst. Tech. J., vol. 55, Apr. 1976.

[2] J. Tow and N. Sachs, "A DSP implementation of digital tone receiver," in Conf. Rec. Int. Conf. Commun., Denver CO, June 1981.

[3] D. C. Rife and R. R. Boorstyn, "Single-tone parameter estimation from discrete-time observations," IEEE Trans. Inform. Theory, vol. IT-20, Sept. 1974

[4] V. K. Jain, "Phase-assisted IPDFT digital DTMF receiver," in Conf. Rec. Int. Conf. Commun., Denver CO, June, 1981.

[5] J. N. Denenberg, "Spectral moment estimator: A new approach to tone detection," Bell Syst. Tech. J., vol. 55, Feb. 1976.

[6] J. G. Gander, "A pattern recognition approach to tone detection," Signal Processing, vol. 1, Jan. 1979.

[7] C. R. Baugh, "Design and performance of a digital multifrequency receiver," IEEE Trans. Commun., vol. COM-25. June 1977.

[8] F. G. Braun, "Nonrecursive digital filters for detecting multifrequency code signals," IEEE Trans. Acoust., Speech, Signal Processing, vol. ASSP-23, June 1975.

[9] G. D. Cain, V. Cappellini, E. Del Re, and L. Fini, "CCD multifrequency-tone receiver with coefficient compensation of chargetransfer loss," in Proc. EUSIPCO 80, Lausanne, Switzerland, Sept. 1980.

[10] J. P. Henry and C. Duret, "Digital multifrequency receiver using nonrecursive (FIR) filters designed with window functions," in Conf. Rec. Int. Conf. Commun., Denver CO, June 1981.

[11] E. Del Re, "A property of digital quadrature filters," Proc. IEEE, vol. 69, Dec. 1981. See also "Corrections to "A property of digital quadrature filters," Proc. IEEE, vol. 70, Aug. 1982

[12] _ "On the performance evaluation of a multifrequency-tone envelope detector," Signal Processing, vol. 3, Jan. 1981.

[13] M. T. McCallig, "Design of digital FIR filters with complex conjugate pulse responses," IEEE Trans. Circuits Syst., vol. CAS-25, Dec. 1978.

[14] E. Del Re and P. L. Emiliani, "An analytic signal approach for transmultiplexers: Theory and design," IEEE Trans. Commun., vol. COM-30, July 1982.

[15] CCITT Green Book, vol. VI(X). Geneva, Switzerland: Int. Telecommun. Union, 1973.

[16] CCITT Green Book, vol. VI(3), ch. 3. Geneva, Switzerland, Int Telecommun. Union, 1973

[17] IEEE DSP Committee, Ed., Programs for Digital Signal Processing. New York: IEEE Press, 1979.

[18] R. W. Schafer and L. R. Rabiner, "A digital signal processing approach to interpolation," Proc. IEEE, vol. 61, June 1973

Vito Cappellini (M'68), for a photograph and biography, see p. 88 of the January 1984 issue of this JouRNAL.

\section{CONCLUSIONS}

An improved structure of a quadrature detection multi-

Enrico Del Re (M'78), for a photograph and biography, see p. 88 of the January 1984 issue of this JournaL. 